\title{
Probiotics as prevention for gastro-intestinal disorders in pediatrics
}

\section{A narrative review}

C. Perceval (1), M. Pletincx (2) Y. Vandenplas (1)

1. KidZ Health Castle, UZ Brussel, Vrije Universiteit Brussel, Brussels, Belgium

2. CHIREC, Ste-Anne St-Remi, Brussels, Belgium

\section{Address for correspondence}

Yvan Vandenplas, KidZ Health Castle, UZ Brussel, Laarbeeklaan 101, 1090 Brussels,

Belgium.

Tel: +324775794; e-mail: yvan.vandenplas@uzbrussel.be 


\section{Abstract}

This is a narrative review, largely of randomized trials on the impacts of probiotics. It concludes that evidence for beneficial effects of selected probiotics in the prevention of gastrointestinal disorders is limited mainly to acute gastroenteritis, antibiotic-associated diarrhea, infantile colic and necrotizing enterocolitis. However, there is no broad consensus to recommend the use of probiotics in the prevention of these conditions, mainly because of the different designs used in different studies, resulting in limited evidence for specific strains, dosages and indications. More well-designed studies utilizing standardized methodologies are needed before recommendations can be proposed. At this stage, there is insufficient evidence to recommend the routine use of probiotics in infants and children for the prevention of gastro-intestinal disorders.

\section{Key Points}

- Data indicate that selected probiotic strains are likely to prevent acute gastroenteritis, antibiotic-associated diarrhea, infantile colic and necrotizing enterocolitis.

- $\quad$ However, relevant studies differ in design.

- $\quad$ As a consequence, there is insufficient evidence for a global recommendation.

- $\quad$ Since adverse effects are extremely rare, one might also recommend the products that were shown beneficial in the above-mentioned indications, considering that patients may only profit, since "there is no harm and since there may be some benefit." 


\section{Introduction}

The microbiome is the totality of all the microbial cells that colonize the human body and their genes. The microbiota genes are far more predominant than the human genome [1]. A balanced microbiome is associated with eubiosis and health, while an unbalanced microbiome or dysbiosis is related to health problems, within and outside the gastro-intestinal (GI) tract. A lot of research is done on how to manipulate the gut microbiome to treat disease and improve human health. Diarrheal illness is the main one examined; it is the second leading cause of mortality among children younger than five years worldwide, causing an estimated 1.5 to 2 million deaths annually. On average, every child under the age of 3 years is reported to develop at least one episode of infectious gastroenteritis per year [2].

The gut microbiota can be altered by medications such as antibiotics and proton pump inhibitors, but also by probiotic supplements. Probiotics are live microorganisms, which when administered in adequate amounts, confer a health benefit on the host [3]. While some authors have published strong evidence to support general effects of probiotics as a group rather than focusing on strain specific effects, others question this approach and yet conclude that there is insufficient evidence to guide the selection of the most effective strains for any specific purpose [4-6]. The aim of this review was to review recent literature regarding the evidence for a health benefit of probiotic administration in the prevention of GI disease in infants and children.

\section{Search strategy and selection criteria}

The following data-bases were searched for randomized controlled trials between Jan 1, 2000 and April 30, 2019.: The Cochrane Library, MEDLINE, and EMBASE. Search terms used were: "probiotics" and/or "prevention” and/or "prophylaxis” and/or "prophylactic use” and "gastrointestinal disorder" and/or "gastrointestinal disease” and “infant” and/or "child” and/or “pediatric”. Languages selected were “English”. 


\section{Probiotics and prevention of diarrhea}

\section{Acute gastroenteritis}

Acute gastroenteritis (AGE) is one of the most frequent infectious diseases during early childhood. The effect of the administration of probiotics has been tested in the prevention of AGE.

In a RCT carried out in residential care settings, Bifidobacterium (B.) lactis Bb 12, when added to an acidified infant formula, was shown to have some, albeit very modest, protective effect against acute diarrhea in healthy children (Table 1) [7]. The difference in the incidence of diarrhea during the study was not statistically different in the probiotic supplemented and control group (28.3 vs 38.7\%). The number of days with diarrhea did not differ between the groups. Feeding infants with the $B$. lactis $B B 12$ reduced the risk of getting diarrhea by a factor of 1.9 [7]. In another RCT, B. animalis subsp. lactis BB-12 given over a period of 3 months had no preventive effect on GI and respiratory tract infections in healthy children who attend day care centers. Overall, the impact on the incidence of diarrhea was not significant [8]. In a community based double-masked, randomized controlled trial in India of children 1-3 years of age who were randomly allocated to receive either control milk or the same milk fortified with 2.4 g/day of prebiotic oligosaccharide and $1.9 \times 10^{7}$ CFU/day of the probiotic B. lactis HN019, there was a significant reduction in dysentery, respiratory morbidity, and febrile illness [9]. In another RCT, daily administration of a combination of B. animalis subsp lactis BB12 and Lactobacillus (L) rhamnosus $G G$ ( $L G G$ ) for 6 months in healthy infants did not reduce the number of episodes of diarrhea, or the number of days the child was absent from child care [10]. In a multicenter trial, infant formulae containing B. lactis and galacto- and fructooligosaccharides (GOS/FOS) did not reduce infection rates compared to formulas with only $B$. lactis [11].

A placebo-controlled trial with $L G G$ showed a decreased incidence of diarrhea in undernourished formula-fed children in Peru, but not in breastfed children [12]. It is tempting to hypothesize that the difference in GI microbiota development in breastfed vs formula fed infants may in part explain this observation. But breastmilk is also a source of protective IgA antibodies [13], which, along with several other factors, protect the infant from developing infectious diarrhea. 
Outcomes in prevention may differ from outcomes in treatment, since two recent therapeutic trials concluded that probiotics (a mixture of L. rhamnosus R0011 and L. helveticus R0052, and $L G G)$ did not shorten the duration of acute gastroenteritis $[14,15]$.

The incidence of diarrhea was significantly reduced with a fermented milk supplement containing L. casei DN-114 001 (15.9\%) compared with yogurt (22.0\%) [16]. Child care infants fed a formula supplemented with L reuteri (American Type Culture Collection 55730) or B. lactis BB 12 had fewer and shorter episodes of diarrhea than children fed regular formula, with no effect on respiratory illnesses [17]. Healthy children attending day care centers, with daily administration of $L$. reuteri DSM 17938 showed a significant effect in reduced episodes and durations of diarrhea and respiratory tract infection compared to placebo, with consequent cost savings for the community [18]. The number of doctor visits, antibiotic use, absenteeism from day school and parental absenteeism from work were significantly reduced in the L. reuteri group $(\mathrm{P}<.05)$ [18]. According to a review, L. reuteri is reported to be effective in reducing the incidence of diarrhea in children attending day care centers [19].

Should administration of probiotics to prevent AGE be recommended? Evidence from literature is limited and differs in design, strains administered, and outcomes measured. Preventive administration of some specific probiotic strains seems to decrease the incidence of AGE-although there are also negative trials $[7,8]$ in regions with a very high incidence of the condition.

\section{Nosocomial diarrhea}

In 1994, the first report that showed a benefit of supplementation of infant formula with $B$. bifidum and Streptococcus thermophilus in reducing the incidence of acute diarrhea and rotavirus shedding in infants admitted to a chronic medical care hospital (Table 2) was published by Saavedra et al. [20] In contrast, B. animalis subsp. lactis BB12 was not effective in preventing nosocomial infections when given to children of more than 1 year during an acute hospitalization [21].

Data regarding $L G G$ are contradictory. Prophylactic use of $L G G$ was shown significantly to reduce the risk of nosocomial diarrhea in infants, particularly nosocomial rotavirus gastroenteritis, resulting in a number needed to treat of 4 [22]. But, formula supplementation with $L G G$ appeared ineffective in preventing nosocomial rotavirus infections, whereas breastfeeding was effective [23]. A randomized controlled trial showed that $L G G\left(6 \times 10^{9}\right.$ colony 
forming units (CFU)/day) together with vitamins $\mathrm{B}$ and $\mathrm{C}$ and zinc given for 15 days, starting on the first day of hospitalization, to children ranging from $0.5-5.0$ years of age resulted in a reduced incidence of nosocomial infections [24].

According to a review, administration of $L G G$ and B. bifidum and Streptococcus thermophilus compared with placebo reduced the risk of healthcare-associated diarrhea [25]. Administration of two other probiotics (L. reuteri DSM 17938 and L. delbrueckii H2B20) was ineffective [25]. Currently there is sufficient evidence showing that $L G G$ administrated in a dose of at least $10^{9}$ CFU/day during a hospital stay can significantly reduce the risk for nosocomial diarrhea in a regular pediatric ward [26]. So far, research has found no evidence of effectiveness of L. reuteri DSM 17938 in preventing nosocomial diarrhea in children $[27,28]$. Based on currently available evidence, there is evidence to recommend $L G G$ when the use of probiotics for preventing nosocomial diarrhea in children is considered, as recommended by the Working Group on Probiotics from the European Society of Paediatric Gastroenterology, Hepatology and Nutrition (ESPGHAN) [29,30].

\section{Antibiotic associated diarrhea}

The prevention of antibiotic associated diarrhea (AAD) has been the subject of many investigations, both in children and adults. Most commonly used probiotics are $L G G, L$. acidophilus, L. casei, B. ssp, Streptococcus ssp, and the yeast Saccharomyces boulardii (S. boulardii). In general, most of these trials do show clear evidence of efficacy, with the two most effective strains being $L G G$ and $S$. boulardii. Evidence is also emerging on the importance of the dose in reducing the incidence of this type of diarrhea, as well as the incidence of Clostridium difficile (C. difficile)-associated post-antibiotic diarrhea [31]. A yogurt combination of $L G G, L$. acidophilus and $B$. BB12 was reported to be an effective method to reduce the incidence of AAD in children (Table 3) [32]. L. plantarum DSM9843 was not better than placebo regarding the incidence of loose/watery stools, mean number of loose/watery stools, or the incidence of abdominal symptoms during antibiotic administration [33]. L. reuteri DSM 17938 was not effective in the prevention of diarrhea or AAD in children [34]. S. boulardii was shown to prevent AAD in children hospitalized because of a respiratory tract infection, and to be effective in the treatment of AAD in children that developed it in the placebo group [35].

According to a review, moderate-quality evidence suggests that probiotics are associated with lower rates of AAD in children (aged 1 month to 18 years) without an increase in adverse events 
[36]. A Cochrane systematic review, analyzing data from 23 studies (3938 participants), estimates a pooled probiotic effect (RR 0.46; 95\% CI: 0.35-0.61) with a number needed to treat of 10 [37]. A post hoc subgroup analysis to explore heterogeneity indicated that probiotics are effective among trials with a $C$. difficile associated diarrhea baseline risk $>5 \%$. The weakness of this kind of meta-analysis is that all probiotic strains are grouped together, while some strains might be more effective than others. Among the various probiotics evaluated, $L G G$ or $S$. boulardii at 5-40 x $10^{9} \mathrm{CFU} /$ day may be appropriate given the modest number needed to treat and the likelihood that adverse events are very rare [33]. In a meta-analysis, LGG was reported to be effective in preventing $\mathrm{AAD}$ in children and adults treated with antibiotics for any reason, although with a moderate to low quality of evidence [38]. Moderate quality evidence suggests that probiotics are associated with a lower risk of $C$. difficile infection and very-low quality evidence suggests that probiotics are associated with fewer adverse events than either placebo or no treatment [39]. ESPGHAN recommends that, if the use of probiotics to prevent AAD is considered because of the existence of risk factors such as class of antibiotic(s), duration of antibiotic treatment, age, need for hospitalization, comorbidities, or previous episodes of AAD diarrhea, LGG (moderate Quality of Evidence (QoE), strong recommendation) or S. boulardii (moderate QoE, strong recommendation) should be used [40]. LGG survival is sensitive to penicillin which might make this probiotic ineffective in when this type of antibiotic is in use [41].

A Cochrane analysis included 33 studies with 6352 participants, assessing the following probiotics: Bacillus spp., Bifidobacterium spp., Clostridium butyricum, Lactobacilli spp., Lactococcus spp., Leuconostoc cremoris, Saccharomyces spp., or Streptococcus spp., alone or in combination [42BB]. The overall evidence suggests a moderate protective effect of probiotics for preventing AAD. The number needed to treat for an additional beneficial outcome was 9 (95\% CI 7 to 13) [42BB]. If only studies with high doses of probiotics are considered ( $\geq$ 5 billion CFUs per day), the number needed to treat for an additional beneficial outcome to prevent one case of diarrhea is reduced to 6 (95\% CI 5 to 9) [42BB].

If the use of probiotics to prevent $C$. difficile-associated diarrhea is considered, $S$. boulardii (low QoE, conditional recommendation) is recommended [30,40]. Other strains or combinations of strains have been tested for this purpose, but evidence for efficacy is insufficient [40]. Despite the need for further research, hospitalized patients, particularly those at high risk of $C$. difficile associated diarrhea, should be informed of the potential benefits and harms of probiotics [30]. S. boulardii, and more recently fecal microbiota transplantation have 
become valid forms of prevention and/or therapy for C. difficile colitis [43]. Analysis has shown that the potential for using $S$. boulardii as AAD prophylactic treatment in adult hospitalized patients in Belgium would, based on 831,655 hospitalizations with antibiotic administration in 2014, result in a $€ 50.3$ cost saving per patient [44]. Generalized use of S. boulardii in hospitalized adults treated with antibiotics could result in total annual savings up to $€ 41.8$ million for the Belgian health care [44]. There are no data on the economic impact of prophylactic probiotic administration to prevent AAD in children.

\section{Probiotics and prevention of infantile colic}

Infantile colic describes excessive crying of unknown cause in otherwise well infants [45]. The incidence is approximately $10 \%$ to $40 \%$ of infants worldwide and is similar among formula-fed and breast-fed infants. Proposed causes include alterations in fecal microbiota, allergy to cow's milk protein, lactose malabsorption, gastrointestinal immaturity or inflammation, increased serotonin secretion, poor feeding technique, and maternal smoking or nicotine replacement therapy [46]. The vast majority of published articles concerning treatment of infantile colic have evaluated probiotics as a therapeutic tool and have shown that L. reuteri DSM 17938 was effective in reducing infantile colic mainly in breastfed infants [47]. Six studies included for subgroup meta-analysis on probiotic treatment, notably L. reuteri, demonstrated that probiotics appear an effective treatment, with an overall mean difference in crying time at day 21 of -55.8 $\min /$ day $(95 \%$ CI -64.4 to $-47.3, \mathrm{P}=0.001)$ [48].

Only limited data are available regarding the use of probiotics in the prevention of this common entity in infancy, since only two clinical studies have been published. The first trial included 468 infants, breastfed as well as formula-fed, revealing that compared with placebo, the daily administration of $L$. reuteri DSM 17938, from day 3 for 90 days, resulted in a significant reduction in crying time by approximately 51 minutes per day at 1 month, and by 33 minutes per day at 3 months. There were also significantly less emergency room visits, lost parental working days and use of additional medications in infants who received the probiotic agent. A cost-benefit analysis revealed significant savings as well [49]. Although almost half of the infants were breast fed, results are not given separately for breast or formula fed infants. Preventive administration of $L$. reuteri was shown to reduce the number of consultations because of colic, and to reduce health care cost, both for the family (88 $€$ ) and for the community $(104 €)[50,51]$. The second study was based on a secondary analysis of data from a trial of $L G G$ supplementation, for the first 6 months of life in 184 infants. No significant differences 
were found between the infants exposed to early $L G G$ supplementation, versus infants exposed to the control intervention [52]. In a third small study, with poorly-defined methods, preventive administration of $B$. breve B632 and BR03 resulted in a mean duration of crying of 12.14 minutes on average in the probiotics group and of 46.65 minutes in the placebo group during the third month of supplementation. However, no significant differences were noticed during the first or second months of supplementation [53]. In view of these conflicting results, further controlled large-scale strain-specific trials are warranted. L. reuteri DSM17938 has been recommended at a dose of $10^{8} \mathrm{CFU}$ once daily as preventive strategy of infantile colic (level I evidence) [30].

A Cochrane review including six studies with 1886 participants, compared probiotics with placebo: two studies examined L. reuteri DSM 17938, two examined multi-strain probiotics, one examined L. rhamnosus, and one examined L.paracasei and B.animalis [54AA]. No clear evidence could be found that probiotics are more effective than placebo at preventing infantile colic; however, daily crying time appeared to reduce with probiotic use compared to placebo [54AA]. In summary, although there is insufficient evidence for a recommendation, available data suggest that specific probiotics strains such as L. reuteri DSM 17938 may prevent infantile colic in some infants. Since $L$ reuteri administration is reported to be safe, the major issue of concern is the cost-benefit.

\section{Probiotics and prevention of necrotizing enterocolitis (NEC)}

NEC is in some countries among the most common and devastating diseases in neonates and thus has become a priority for research [55]. The pathophysiology of classic NEC is incompletely understood, but epidemiologic observations strongly suggest a multifactorial cause [56]. Inappropriate initial microbial colonization in preterm infants is considered to be an important risk factor for NEC [57], particularly since NEC does not occur until at least 8 to 10 days postpartum, at a time when anaerobic bacteria have colonized the gut. Furthermore, experimental NEC does not occur in germ-free animals [58], and infants with NEC frequently have concomitant bacteremia and endotoxemia [59]. C. perfringens is associated with NEC from the first meconium till just before NEC onset [60]. In contrast, post-meconium, increased numbers of staphylococci were negatively associated with NEC [60].

L. reuteri DSM 17938 administered to preterm infants was shown to be safe and to reduce significantly feeding intolerance [61]. No significant differences were found for any other secondary outcomes such as necrotizing enterocolitis (NEC), hospital stay, sepsis and diarrhea. 
In contrast, a meta-analysis concluded that bifidobacteria administration reduced the relative risk of developing NEC (RR 0.38, 95\% CI 0.25-0.58; P < 0.00001) or death (RR 0.74, 95\% CI $0.60-0.92 ; \mathrm{P}=0.006$ ), but no significant difference in the incidence of sepsis was found (RR 0.87, 95\% CI 0.73-1.03; $\mathrm{P}=0.11$ ) [62]. In a retrospective observational study, the incidence of NEC in 640 very low birth weight infants with a median gestational age of 28.7 weeks that were given LGG was $12 \%$ compared to $10.2 \%$ before the implementation of the probiotic administration [63]. The conclusion of this trial was that $L G G$ increased the risk to develop NEC [63]. However, another group came to an opposite conclusion with a comparable protocol in a retrospective observational study performed in a resource limited setting: $L G G$ reduced significantly NEC $\geq$ Stage II and the composite outcome of NEC $\geq$ Stage II/mortality in preterm infants [64]. According to a strain-specific network meta-analysis, only 3 of 25 studied probiotic treatment combinations (the combination of B.bifidum NCDO 1453 and $L$. acidophilus NCDO 1748 (based on 2 studies with 494 infants); the combination of B. bifidum, B. infantis, B. longum, and L. acidophilus (based on 1 study with 186 infants); and the combination of B. infantis, L. acidophilus, L. casei, L. plantarum, L. rhamnosus, and S. thermophilus altogether (based on 1 study with 150 infants) showed significant reduction in mortality [64]. Seven treatments reduced NEC incidence (B.lactis Bb-12 or B94, based on 5 trials with 828 infants; L. reuteri ATCC 55730 or DSM 17938, based on 4 studies with 1459 infants; L. GG, based on 6 studies with 1507 infants); the combination of B. bifidum, B. infantis, B. longum, and L. acidophilus, based on 2 studies with 247 infants; the combination of $B$. infantis ATCC 15697 and L. acidophilus ATCC 4356, based on one study with 367 infants; the combination of B. infantis Bb-02, B. lactis Bb-12, and S. thermophilus TH-4, based on 2 studies with 1244 infants; and the combination of B.longum 35624 and $L G G$, based on 2 studies with 285 infants, 2 reduced late-onset sepsis (combination of B. bifidum, B. infantis, B. longum, and L. acidophilus (based on 2 studies with 247 infants); for the combination of B. longum R00175, L. helveticus R0052, L. rhamnosus R0011, and S. Boulardii CNCM I-1079, based on 3 studies with 241 infants, and 3 reduced time until full enteral feeding (L. reuteri ATCC 55730 or DSM 17938, based on 3 studies with 626 infants); for the combination of B. bifidum, B. infantis, $B$. longum, and L.acidophilus, based on 2 studies with 247 infants; and for the combination of $B$. longum BB536 and LGG, based on 1 study with 94 infants [64]. There was no clear overlap of strains, which were effective on multiple outcome domains [64]. The network meta-analysis showed efficacy in reducing mortality and morbidity in only a minority of the studied strains or combinations. This may be due to an inadequate number or size of randomized controlled trials, or due to a true lack of effect for certain species [65]. The importance of strain specificity 
and a demonstration of safety is highlighted since a specific product (Infloran ${ }^{\mathrm{TM}}$ ) was reported to increase the incidence of NEC [66]. Further large and adequately powered randomized controlled trials using strains with the greatest apparent efficacy will be needed to define more precisely optimal treatment strategies.

Compared to formula feeding, breastmilk protects for NEC. However, both in breast- and formula-fed preterms probiotics seem to be one of the most significant advances in NEC prevention at present because of the significant range of beneficial effects at various levels of gut function and defense mechanisms [4,30]. While some authors published strong evidence to support general effects of probiotics as a group, rather than focusing on strain specific effects, others do question this approach and conclude that there is insufficient evidence to guide the selection of the most effective strains [4-6].

\section{Probiotics and prevention of regurgitation}

Regurgitation is one of the most common functional gastrointestinal disorders in infants, with a significant impact on quality of life of the infants and the family $[67,68]$. Administration of L. reuteri DSM 17938 prevented regurgitation episodes during the first month of life in exclusively breastfed infants, when compared to historic controls [69,70]. Prophylactic use of L. reuteri DSM 17938 from birth to 3 months resulted in a decreased number of episodes of regurgitations per day, compared to no probiotic ( 2.9 vs $4.6 ; \mathrm{P}<.01)$ [49]. This finding is likely to be related to the faster gastric emptying induced by the probiotic [70]. A synbiotic infant formula, supplemented with $B$. lactis and fructo-oligosaccharides, with lactose and a whey/casein 60/40 protein ratio was tested in 280 infants over 3 months and resulted in a lower incidence of daily regurgitation (10.9\% of all infants) compared to the median prevalence for a similar age according to historic data from literature (median value of 26.7\%) [71]. Some probiotic strains may enhance gastric emptying and therefore have a beneficial effect on functional gastro-intestinal symptoms of the esophagus and stomach.

L. reuteri DSM 17938 decreased dysbiosis in children treated with proton pump inhibitors [72]. After 12 weeks of treatment with a proton pump inhibitor, dysbiosis was diagnosed according to the results of a glucose hydrogen breath test in $56.2 \%$ of the children in the placebo group, compared to $6.2 \%$ of the children in the probiotic group $(\mathrm{P}<0.001)$ [72]. Bacterial overgrowth was detected in $5 \%$ of controls, which is similar to the group treated with L. reuteri and proton pump inhibitors [72]. 
There is insufficient evidence from literature to recommend routine administration of some specific probiotic strains for the prevention of regurgitation. However, no study suggested that probiotics may increase the risk for regurgitation. L. reuteri DSM 17938 may decrease the adverse effects of proton pump inhibitors on the GI microbiota.

\section{Probiotics and prevention of constipation}

A meta-analysis concluded that there is insufficient evidence to recommend pre-, pro- or synbiotics in the treatment of children with functional constipation [73]. Another meta-analysis showed that some probiotic strains increase stool frequency in Asian children [74]. A synbiotic infant formula, supplemented with B. lactis and fructo-oligosaccharides, was tested in 280 infants over a 3-month period and showed a lower incidence of constipation (3.2\%) than the incidence reported in literature (7.8\%) [71]. L. reuteri DSM 17938 administration resulted in a statistically significant increase in mean number of defecations per day compared to placebo in infants (4.2 vs 3.6; $\mathrm{P}<.01$ ) [48]. Although there is insufficient evidence for a recommendation, there are some data that preventive administration of probiotics to infants may increase the number of defecations per day.

\section{Probiotics and prevention of Helicobacter pylori}

Lactobacilli, as an adjunct to triple therapy, increases Helicobacter pylori eradication rates and reduces the incidence of therapy-related diarrhea in children [75]. According a meta-analysis of data obtained with S. boulardii in 11 RCTs (2200 participants, among them 330 children), the yeast probiotic is likely to increase the eradication rate by about 10 percent and to decrease the adverse effects of the eradication therapy [76]. A meta-analysis of 5 studies (434 participants), concluded that the lactobacilli strains differed among studies: L. acidophilus and L. rhamnosus, L. reuteri, L. casei, LGG, and compound lactobacillus but detailed information was rarely provided of the strains used [75]. However, there are no data on the prevention of Helicobacter pylori infection by the administration of probiotics.

\section{Probiotics and small bowel bacterial overgrowth}

There are a few studies in adults showing that the clinical consequence of small intestinal bacterial overgrowth can be treated effectively by administration of probiotics [77]. However, L. rhamnosus R0011 $\left(1.9 \times 10^{9} \mathrm{CFU}\right)$ and L. acidophilus R0052 $\left(0.1 \times 10^{9} \mathrm{CFU}\right)$ failed to decrease the incidence of small bowel bacterial overgrowth in children treated with omeprazole 
[78]. However, we could not find any information on the use of probiotics in the prevention of this condition.

\section{Probiotics and prevention of irritable bowel syndrome}

Although there are some data that some specific strains alleviate pain in children with irritable bowel syndrome $[79,80]$, we could not find information on prevention.

\section{Probiotics and prevention of inflammatory bowel disease}

No randomized controlled trials were found evaluating if preventive administration of probiotics may decrease the number of flares of inflammatory bowel disease in children.

\section{Conclusions}

The authors of this review strongly believe in strain and product specificity in probiotic research. Extrapolation from studied strains to unstudied strains and products could lead to erroneous conclusions. Clinical trials using commercialized products should give attention to influencing factors such as product quality and shelf life.

The ability to impact the microbiome with probiotics is an interesting approach in the prevention of GI diseases, but studies on probiotic administration to prevent GI disorders are limited. Most studies focus on treatment and not prevention. The studies available on prevention of gastro-intestinal diseases in children focus on infectious, nosocomial and antibioticassociated diarrhea or NEC, and there are some studies on infantile colic.

Studies on the prevention of NEC differ in design and strains tested. Partly for this reason, there is no consensus to recommend the routine administration of probiotics to preterm infants to prevent NEC. The possibility of serious adverse effects in preterm infants should also be considered in continuing research.

There is also no consensus if probiotics should be administered routinely to normal infants to prevent acute gastroenteritis, AAD and infantile colic. The best evidence for benefit regards $B$. lactis (for acute gastroenteritis), S. boulardii and LGG (for AAD) and L. reuteri DSM 17938 for infantile colic, for regurgitation and stool composition. Despite the lack of evidence, many infant formulae do contain probiotics and thus many infants are exposed to daily intake of probiotic strains. Research is inadequate to judge whether or not to recommend the use of these products in artificially fed infants. 
Overall there are insufficient data to recommend routine administration of probiotics to prevent GI disorders. However, one could also consider that preventive probiotic administration is unlikely to be harmful or cause adverse effects except possibly in very vulnerable infants such as prematures and that preventive administration of probiotics can be considered because of the safety profile even if the evidence suggesting benefit is limited so far.

\section{References}

1. Cani PD (2018). Human gut microbiome: hopes, threats and promises. Gut67: 1716-1725

2. Lo Vecchio A, Vandenplas Y, Benninga Ml (2016). An international consensus report on a new algorithm for the management of infant diarrhoea. Acta Paediatr. 105: e384-389

3. Hill C, Guarner F, Reid G (2014). The International Scientific Association for Probiotics and Prebiotics consensus statement on the scope and appropriate use of the term probiotic. Nat Rev Gastroenterol Hepatol. 11:506-514

4. van den Akker CHP, van Goudoever JB, Szajewska H (2018). Probiotics for preterm infants: a strain-specific systematic review and network meta-analysis. J Pediatr Gastroenterol Nutr. 67:103-122

5. Pell LG, Loutet MG, Roth DE (2019). Arguments against routine administration of probiotics for NEC prevention. Curr Opin Pediatr. 31:195-201

6. Sanders ME, Benson A, Lebeer S (2018). Shared mechanisms among probiotic taxa: implications for general probiotic claims. Curr Opin Biotechnol. 49:207-216

7. Chouraqui JP, Van Egroo LD, Fichot MC (2004) Acidified milk formula supplemented with bifidobacterium lactis: impact on infant diarrhea in residential care settings. J Pediatr Gastroenterol Nutr;38:288-292

8. Hojsak I, Močić Pavić A, Kos T (2016). Bifidobacterium animalis subsp. lactis in prevention of common infections in healthy children attending day care centers - Randomized, double blind, placebo-controlled study. Clin Nutr 35:587-591

9. Sazawal S, Dhingra U, Hiremath G (2010). Prebiotic and probiotic fortified milk in prevention of morbidities among children: community-based, randomized, double-blind, controlled trial. PLoS One 5:e12164 
10. Laursen RP, Larnkjær A, Ritz C (2017). Probiotics and child care absence due to infections: a randomized controlled trial. Pediatrics140:pii:e2017073.

11. Bocquet A, Lachambre E, Kempf C (2013). Effect of infant and follow-on formulas containing B lactis and galacto- and fructo-oligosaccharides on infection in healthy term infants. J Pediatr Gastroenterol Nutr. 57:180-187

12. Oberhelman RA, Gilman RH, Sheen P (1999). A placebo-controlled trial of Lactobacillus GG to prevent diarrhea in undernourished Peruvian children. J Pediatr 134:15-20.

13. Schneider C, Illi M, Lötscher M (2017). Isolation of Antibodies from Human Plasma, Saliva, Breast Milk, and Gastrointestinal Fluid. Methods Mol Biol. 1643: 23-31

14. Freedman SB, Williamson-Urquhart S, Farion KJ (2018). Multicenter trial of a combination probiotic for children with gastroenteritis. N Engl J Med.379:2015-2026

15. Schnadower D, Tarr PI, Casper TC (2018). Lactobacillus rhamnosus GG versus placebo for acute gastroenteritis in children. N Engl J Med. 379:2002-2014

16. Pedone CA, Arnaud CC, Postaire ER (2000). Multicentric study of the effect of milk fermented by Lactobacillus casei on the incidence of diarrhoea. Int J Clin Pract. 54:568-571

17. Weizman Z, Asli G, Alsheikh A (2005). Effect of a probiotic infant formula on infections in child care centers: comparison of two probiotic agents. Pediatrics. 115:5-9.

17. Gutierrez-Castrellon P, Lopez-Velazquez G, Diaz-Garcia L (2014). Diarrhea in preschool children and Lactobacillus reuteri: a randomized controlled trial. Pediatrics 133:e904-9.

19. Urbańska M, Szajewska H (2014). The efficacy of Lactobacillus reuteri DSM 17938 in infants and children: a review of the current evidence. Eur J Pediatr. 2173:1327-1337

20. Saavedra JM, Bauman NA, Oung I (1994). Feeding of Bifidobacterium bifidum and Streptococcus thermophilus to infants in hospital for prevention of diarrhoea and shedding of rotavirus. Lancet 344:1046-1049

21. Hojsak I, Tokić Pivac V, Močić Pavić A (2015). Bifidobacterium animalis subsp. lactis fails to prevent common infections in hospitalized children: a randomized, double-blind, placebocontrolled study. Am J Clin Nutr 101:680-684 
22. Szajewska H, Kotowska M, Mrukowicz JZ (2001). Efficacy of Lactobacillus GG in prevention of nosocomial diarrhea in infants. J Pediatr 138:361-365.

23. Mastretta E, Longo P, Laccisaglia A, Balbo L (2002). Effect of Lactobacillus GG and breast-feeding in the prevention of rotavirus nosocomial infection. J Pediatr Gastroenterol Nutr 35:527-531

24. Bruzzese E, Fedele MC, Bruzzese D (2016). Randomised clinical trial: a Lactobacillus GG and micronutrient-containing mixture is effective in reducing nosocomial infections in children, vs. placebo. Aliment Pharmacol Ther 44:568-575.

25. Wanke M, Szajeweska H (2014). Probiotics for preventing healthcare-associated diarrhea in children: A meta-analysis of randomized controlled trials. Pediatr Pol 89:8-16.

26. Trivić I, Hojsak I (2018). Use of probiotics in the prevention of nosocomial infections. J Clin Gastroenterol 52 Suppl 1:S62-65.

27. Urbańska M, Gieruszczak-Białek D, Szymański H (2016). Effectiveness of Lactobacillus reuteri DSM 17938 for the prevention of nosocomial diarrhea in children: A randomized, double-blind, placebo-controlled trial. Pediatr Infect Dis J 35:142-145.

28. Wanke M, Szajewska H (2012). Lack of an effect of Lactobacillus reuteri DSM 17938 in preventing nosocomial diarrhea in children: a randomized, double-blind, placebo-controlled trial. J Pediatr 161:40-43.

29. Hojsak I, Szajewska H, Canani RB (2018). Probiotics for the Prevention of nosocomial diarrhea in children. J Pediatr Gastroenterol Nutr 66:3-9.

30. Cameron D, Hock QS, Kadim M (2017). Probiotics for gastrointestinal disorders: Proposed recommendations for children of the Asia-Pacific region. World J Gastroenterol 23:7952-796.4

31. Guandalini S (2011) Probiotics for prevention and treatment of diarrhea. J Clin Gastroenterol. 45:S149-153.

32. Fox MJ, Ahuja KD, Robertson IK (2015). Can probiotic yogurt prevent diarrhoea in children on antibiotics? A double-blind, randomised, placebo-controlled study. BMJ Open. 5:e006474. 
33. Olek A, Woynarowski M, Ahrén IL (2017). Efficacy and safety of Lactobacillus plantarum DSM 9843 (LP299V) in the prevention of antibiotic-associated gastrointestinal symptoms in children-randomized, double-blind, placebo-controlled study. J Pediatr. 186:82-86.

34. Kołodziej M, Szajewska H (2018). Lactobacillus reuteri DSM 17938 in the prevention of antibiotic-associated diarrhoea in children: a randomized clinical trial. Clin Microbiol Infect. pii:S1198-743X(18)30591-3.

35. Shan LS, Hou P, Wang ZJ (2013). Prevention and treatment of diarrhoea with Saccharomyces boulardii in children with acute lower respiratory tract infections. Benef Microbes. 4:329-334.

36. Johnston BC, Goldenberg JZ, Parkin PC (2016). Probiotics and the prevention of antibioticassociated diarrhea in infants and children. JAMA. 316:1484-1485.

37. Goldenberg JZ, Lytvyn L, Steurich J (2015). Probiotics for the prevention of pediatric antibiotic-associated diarrhea. Cochrane Database Syst Rev. 12:CD004827.

38. Szajewska H, Kołodziej M (2015) Systematic review with meta-analysis: Lactobacillus rhamnosus GG in the prevention of antibiotic-associated diarrhoea in children and adults. Aliment Pharmacol Ther. 42:1149-1157.

39. Goldenberg JZ, Mertz D, Johnston BC (2018). Probiotics to prevent Clostridium difficile infection in patients receiving antibiotics. JAMA. 320:499-500.

40. Szajewska H., Canani RB, Guarino A (2016). ESPGHAN Working Group for Probiotics and Prebiotics. Probiotics for the prevention of antibiotic-associated diarrhea in children. $\mathrm{J}$ Pediatr Gastroenterol Nutr. 62:495-506.

41. Mantegazza C, Molinari P, D'Auria E (2018). Probiotics and antibiotic-associated diarrhea in children: A review and new evidence on Lactobacillus rhamnosus GG during and after antibiotic treatment. Pharmacol Res. 128:63-72.

42. Guo Q, Goldenberg JZ, Humphrey C, El Dib R, Johnston BC (2019). Probiotics for the prevention of pediatric antibiotic-associated diarrhea. Cochrane Database Syst Rev. 4:CD004827.

43. Dinleyici M, Vandenplas Y (2019). Clostridium difficile colitis prevention and treatment Adv Exp Med Biol. 1125:139-146. doi: 10.1007/5584_2018_322. 
44. Vermeersch SJ, Vandenplas Y, Tanghe A (2018). Economic evaluation of S. boulardii CNCM I-745 for prevention of antibiotic associated diarrhoea in hospitalized patients. Acta Gastroenterol Belg. 81:269-276.

45. Sung V (2018) Infantile colic. Aust Prescr. 41:105-110.

46. Johnson JD, Cocker K, Chang E (2015). Infantile colic: recognition and treatment. Am Fam Physician. 92:577-582.

47. Sung V, D'Amico F, Cabana MD (2018). Lactobacillus reuteri to treat infant colic: a metaanalysis. Pediatrics. 141:e20171811.

48. Harb T, Matsuyama M, David M (2016). Infant colic-what works: a systematic review of interventions for breast-fed infants. J Pediatr Gastroenterol Nutr. 62:668-686.

49. Indrio F, Di Mauro A, Riezzo G (2014). Prophylactic use of a probiotic in the prevention of colic, regurgitation and functional constipation: a randomized clinical trial. JAMA Pediatr. 168:228-233.

50. Savino F, Ceratto S, Poggi E (2015). Preventive effects of oral probiotic on infantile colic: a prospective, randomised, blinded, controlled trial using Lactobacillus reuteri DSM 17938. Benef Microbes. 6:245-251.

51. Indrio F, Di Mauro A, Riezzo G (2015). Prevention of functional gastrointestinal disorders in neonates: clinical and socioeconomic impact. Benef Microbes. 6:195-198.

52. Cabana MD, McKean M, Beck AL (2019). Pilot analysis of early LGG for infant colic prevention. J Pediatr Gastroenterol Nutr 68:17-19.

53. Giglione E, Prodam F, Bellone S (2016). The association of bifidobacterium breve br03 and b632 is effective to prevent colics in bottle-fed infants: a pilot, controlled, randomized, and double-blind study. J Clin Gastroenterol. 50 Suppl 2:S164-S167.

54. Ong TG, Gordon M, Banks SS, Thomas MR, Akobeng AK (2019). Probiotics to prevent infantile colic. Cochrane Database Syst Rev. 3:CD012473.

55. Grave GD, Nelson SA, Walker WA (2007). New therapies and preventive approaches for necrotizing enterocolitis: report of a research planning workshop. Pediatr Res. 62:510-514.

56. Neu J, Walker WA (2011). Necrotizing enterocolitis. N Engl J Med. 20:255-264. 
57. Claud EC, Walker WA (2001). Hypothesis: inappropriate colonization of the premature intestine can cause neonatal necrotizing enterocolitis. FASEB J. 15:1398-1403.

57. Morowitz MJ, Poroyko V, Caplan M (2010). Redefining the role of intestinal microbes in the pathogenesis of necrotizing enterocolitis. Pediatrics 125:777-785.

58. Schwiertz A, Gruhl B, Löbnitz M (2003). Development of the intestinal bacterial composition in hospitalized preterm infants in comparison with breast-fed, full-term infants. Pediatr Res. 54:393-399.

60. Collado MC, Rautava S, Aakko J (2016). Human gut colonisation may be initiated in utero by distinct microbial communities in the placenta and amniotic fluid. Sci Rep. 6:23129.

61. Forsberg A, West CE, Prescott SL (2016). Pre-and probiotics for allergy prevention: time to revisit recommendations? Clin Exp Allergy 46:1506-1521

62. Zhu XL, Tang XG, Qu F (2019) l. Bifidobacterium may benefit the prevention of necrotizing enterocolitis in preterm infants: A systematic review and meta-analysis. Int J Surg. $61: 17-25$.

63. Heida FH, van Zoonen AG, Hulscher JB (2016). A necrotizing enterocolitis-associated gut microbiota is present in the meconium: results of a prospective study. Clin Infect Dis. 62:863870.

64. Kane AF, Bhatia AD, Denning PW (2018). Routine supplementation of Lactobacillus rhamnosus GG and risk of necrotizing enterocolitis in very low birth weight infants. J Pediatr. 195:73-79.

65. Patel S, Chaudhari M, Kadam S (2018). Standardized feeding and probiotic supplementation for reducing necrotizing enterocolitis in preterm infants in a resource limited set up. Eur J Clin Nutr. 72:281-287.

66. Escribano E, Zozaya C, Madero R (2018). Increased incidence of necrotizing enterocolitis associated with routine administration of Infloran ${ }^{\mathrm{TM}}$ in extremely preterm infants. Benef Microbes 9:683-690 
67. Vandenplas Y, Hauser B, Salvatore S (2019). Functional gastrointestinal disorders in infancy: impact on the health of the infant and family. Pediatr Gastroenterol Hepatol Nutr. 22:207-216.

68. Salvatore S, Abkari A, Cai W, Catto-Smith A, Cruchet S, Gottrand F, Hegar B, Lifschitz C, Ludwig T, Shah N, Staiano A, Szajewska H1 Treepongkaruna S, Vandenplas Y (2018). Review shows that parental reassurance and nutritional advice help to optimise the management of functional gastrointestinal disorders in infants. Acta Paediatr. 2018 107:1512-1520

69. Garofoli F, Civardi E, Indrio F (2014). The early administration of Lactobacillus reuteri DSM 17938 controls regurgitation episodes in full-term breastfed infants. Int J Food Sci Nutr. 65:646-648.

70. Indrio F, Riezzo G, Raimondi F (2011). Lactobacillus reuteri accelerates gastric emptying and improves regurgitation in infants. Eur J Clin Invest. 41:417-422.

71. Vandenplas Y, Analitis A, Tziouvara C (2017). Safety of a new synbiotic starter formula. Pediatr Gastroenterol Hepatol Nutr. 20:167-177.

72. Belei O, Olariu L, Dobrescu A (2018). Is it useful to administer probiotics together with proton pump inhibitors in children with gastroesophageal reflux? J Neurogastroenterol Motil. 24:51-57.

73. Koppen IJ, Benninga MA, Tabbers MM (2016). Is there a role for pre-, pro- and synbiotics in the treatment of functional constipation in children? A systematic review. J Pediatr Gastroenterol Nutr. 63 Suppl 1:S27-35.

74. Huang R, Hu J (2017). Positive effect of probiotics on constipation in children: a systematic review and meta-analysis of six randomized controlled trials. Front Cell Infect Microbiol. $7: 153$.

75. Fang HR, Zhang GQ, Cheng JY, Li ZY (2019). Efficacy of Lactobacillus-supplemented triple therapy for Helicobacter pylori infection in children: a meta-analysis of randomized controlled trials. Eur J Pediatr. 178:7-16.

76. Szajewska H, Horvath A, Kołodziej M (2015). Systematic review with meta-analysis: Saccharomyces boulardii supplementation and eradication of Helicobacter pylori infection. Aliment Pharmacol Ther. 41:1237-1245. 
77. Gabrielli M, Lauritano EC, Scarpellini E, Lupascu A, Ojetti V, Gasbarrini G, Silveri NG, Gasbarrini A (2009). Bacillus clausii as a treatment of small intestinal bacterial overgrowth. Am J Gastroenterol. 104:1327-1328.

78. Hegar B, Hutapea EI, Advani N (2013). A double-blind placebo-controlled randomized trial on probiotics in small bowel bacterial overgrowth in children treated with omeprazole. J Pediatr (Rio J). 89:381-387.

79. Wegh CAM, Benninga MA, Tabbers MM (2018). Effectiveness of probiotics in children with functional abdominal pain disorders and functional constipation: a systematic review. $\mathrm{J}$ Clin Gastroenterol. 52 Suppl 1:S10-S26.

80. Guandalini S, Magazzù G, Chiaro A (2010). VSL\#3 improves symptoms in children with irritable bowel syndrome: a multicenter, randomized, placebo-controlled, double-blind, crossover study. J Pediatr Gastroenterol Nutr. 51:24-30. 
Table 1: Probiotics and prevention of acute gastroenteritis

\begin{tabular}{|c|c|c|c|c|}
\hline Author (year) ref & Strain & $\begin{array}{l}\text { Incide } \\
\text { gastro }\end{array}$ & $\begin{array}{l}\text { e acute } \\
\text { teritis }\end{array}$ & $\mathbf{p}$ \\
\hline & & Probiotic & Placebo & \\
\hline Chouraqui (2004) ${ }^{7}$ & B lactis $B b 12$ & $28.3 \%$ & $38.7 \%$ & NS \\
\hline Hojsak $^{\mathrm{a}}(\mathbf{2 0 1 6})^{8}$ & $B$ animaliss $B b 12$ & $64.4 \%$ & $61.3 \%$ & NS \\
\hline Sazawal (2010) ${ }^{9}$ & $\begin{array}{l}\text { B. lactis HN019 } \\
\text { (+ prebiotic) }\end{array}$ & 5.26 ер & 5.44 ер & NS \\
\hline Laursen (2017) ${ }^{10}$ & $\begin{array}{l}\text { B. lactis BB12 } \\
\text { L. rhamnosus GG }\end{array}$ & $64 \%$ & $56 \%$ & 0.14 \\
\hline Bocquet $^{\mathrm{a}}(2013){ }^{11}$ & B. lactis BB12 & $4.5+3.0$ ер & $4.9^{b}+3.2$ ep & 0.18 \\
\hline Oberhelman (1999) ${ }^{12}$ & L. rhamnosus GG & 5.21 & 6.02 & 0.028 \\
\hline Pedone (2000) ${ }^{16}$ & L. casei DN-114 001 & $15.9 \%$ & $22 \%^{c}$ & 0.03 \\
\hline Weizman (2005) ${ }^{17}$ & $\begin{array}{l}\text { L. reuteri ATC } 55730 \\
\text { B. Lactis BB12 }\end{array}$ & $\begin{array}{l}0.02 \text { ep } \\
0.13 \text { ep }\end{array}$ & 0.31 ер & $<0.001$ \\
\hline${ }^{\mathrm{a}}$ common infections rep & d (not only acute gastro & ateritis) & & \\
\hline brebiotics group & & & & \\
\hline${ }^{c}$ yoghurt & & & & \\
\hline ep=episodes & & & & \\
\hline $\mathrm{B}=$ Bifidobacterium & & & & \\
\hline L. $=$ Lactobacillus & & & & \\
\hline
\end{tabular}


Table 2: Probiotics and prevention of nosocomial diarrhea

\begin{tabular}{|c|c|c|c|c|}
\hline \multirow[t]{2}{*}{ Author (year) ref } & \multirow[t]{2}{*}{ Strains } & \multicolumn{2}{|c|}{$\begin{array}{c}\text { Incidence nosocomial } \\
\text { diarrhea }\end{array}$} & \multirow[t]{2}{*}{$\mathbf{p}$} \\
\hline & & Probiotic & Placebo & \\
\hline Saavedra (1994) ${ }^{20}$ & $\begin{array}{l}\text { B. bifidum } \\
\text { Str. thermophilus }\end{array}$ & $7 \%$ & $31 \%$ & 0.035 \\
\hline Hojsak (2015) ${ }^{21}$ & B. animalis $\mathrm{BB} 12$ & $8.0 \%$ & $6.0 \%$ & NS \\
\hline Szajewska (2001) ${ }^{22}$ & L. rhamnosus GG & $6.7 \%$ & $33.3 \%$ & 0.002 \\
\hline Mastretta (2002) ${ }^{23}$ & L. rhamnosus GG & $25.4 \%$ & $30.2 \%$ & 0.432 \\
\hline Bruzzese (2016) ${ }^{24}$ & L. rhamnosus GG & $9 \%$ & $33 \%$ & 0.016 \\
\hline Urbańska (2016) ${ }^{27}$ & L. reuteri DSM 17938 & $6.4 \%$ & $7.7 \%$ & NS \\
\hline Wanke (2012) ${ }^{28}$ & L. reuteri DSM 17938 & $33 \%$ & $31 \%$ & NS \\
\hline \multicolumn{5}{|l|}{ B.=Bifidobacterium } \\
\hline \multicolumn{5}{|l|}{ Str=Streptococus } \\
\hline L.=Lactobacillus & & & & \\
\hline
\end{tabular}


Table 1: Probiotics and prevention of antibiotic associated diarrhea

\begin{tabular}{|c|c|c|c|c|}
\hline Author (year) ref & Strains & \multicolumn{2}{|c|}{ AAD } & $\mathbf{p}$ \\
\hline & & probiotic & placebo & \\
\hline Fox (2015) ${ }^{32}$ & $\begin{array}{l}\text { L. rhamnosus GG } \\
\text { B. lactis Bb-12 } \\
\text { L. acidophilus La-5 }{ }^{\mathrm{a}}\end{array}$ & $0 \%$ & $18 \%$ & 0.025 \\
\hline Olek (2017) ${ }^{33}$ & L. plantarum DSM9843 & $39 \%$ & $44.5 \%$ & NS \\
\hline Kołodziej (2018) ${ }^{34}$ & L. reuteri DSM 17938 & $6.5 \%$ & $11.4 \%$ & NS \\
\hline Shan (2013) ${ }^{35}$ & S. boulardii & $4.3 \%$ & $19.4 \%$ & $<0.001$ \\
\hline
\end{tabular}

aboth probiotic and placebo groups received yoghurt;

$\mathrm{AAD}=$ Antibiotic Associated Diarrhea

L.=Lactobacillus

B.=Bifidobacterium

S.=Saccharomyces 